\title{
Implications of lipid monolayer charge characteristics on their selective interactions with a short antimicrobial peptide
}

DOI:

10.1016/j.colsurfb.2016.10.043

\section{Document Version}

Accepted author manuscript

Link to publication record in Manchester Research Explorer

Citation for published version (APA):

Ciumac, D., Campbell, R., Xu, H., Clifton, L., Hughes, A., Webster, J. R. P., \& Lu, J. (2016). Implications of lipid monolayer charge characteristics on their selective interactions with a short antimicrobial peptide. Colloids and Surfaces B: Biointerfaces, 150, 308-316. https://doi.org/10.1016/j.colsurfb.2016.10.043

\section{Published in:}

Colloids and Surfaces B: Biointerfaces

\section{Citing this paper}

Please note that where the full-text provided on Manchester Research Explorer is the Author Accepted Manuscript or Proof version this may differ from the final Published version. If citing, it is advised that you check and use the publisher's definitive version.

\section{General rights}

Copyright and moral rights for the publications made accessible in the Research Explorer are retained by the authors and/or other copyright owners and it is a condition of accessing publications that users recognise and abide by the legal requirements associated with these rights.

\section{Takedown policy}

If you believe that this document breaches copyright please refer to the University of Manchester's Takedown Procedures [http://man.ac.uk/04Y6Bo] or contact uml.scholarlycommunications@manchester.ac.uk providing relevant details, so we can investigate your claim.

\section{OPEN ACCESS}


Implications of lipid monolayer charge characteristics on their selective interactions with a short antimicrobial peptide

Daniela Ciumac, Richard A. Campbell, Hai Xu, Luke A. Clifton, Arwel V. Hughes, John W P Webster and Jian R. Lu

\section{Recount}

Statistical summary: - $\quad$ manuscript (title, keywords, highlights, abstract, main text until author info): 6300 words

- Authors info: 144 words

- References: 567 words

- Figure captions + tables: $\mathbf{4 2 0}$ words (8 equivalent as suggested)

Keywords: neutron reflection, lipid layers, membrane lytic, selectivity, membrane binding, peptides

Highlights: Effects of lipid monolayer charges on the binding of antimicrobial peptides; Structure and composition of lipid monolayers before and after antimicrobial peptide binding; relevance to antimicrobial activity. 


\section{Abstract:}

Many antibacterial peptides (AMPs) target bacterial membranes and they kill bacteria by causing structural disruptions. One of the fundamental issues however lies in the selective responses of AMPs to different cell membranes as a lack of selectivity can elicit toxic side effects to mammalian host cells. A key difference between the outer surfaces of bacterial and mammalian cells is the charge characteristics. We report a careful study of the binding of one of the representative AMPs, with the general sequence $\mathrm{G}(\mathrm{IIKK})_{4} \mathrm{I}-\mathrm{NH}_{2}\left(\mathrm{G}_{4}\right)$, to the spread lipid monolayers of DPPC (1,2-dipalmitoyl-sn-glycero-3-phosphocholine) and DPPG (1,2-dipalmitoylsn-glycero-3-phospho-(1'-rac-glycerol) (sodium salt)) mimicking the charge difference between them, using the combined measurements from Langmuir trough, Brewster angle microscopy (BAM) and neutron reflectivity (NR). The difference in pressure rise upon peptide addition into the subphase clearly demonstrated the different interactions arising from different lipid charge features. Morphological changes from the BAM imaging confirmed the association of the peptide into the lipid monolayers, but there was little difference between them. However, NR studies revealed that the peptide bound 4 times more onto DPPG monolayer than onto the DPPC monolayer. Importantly, whilst the peptide could only be associated with the head groups of DPPC it was well penetrated into the entire DPPG monolayer, showing that the electrostatic interaction strengthened the hydrophobic interaction and that the combined molecular interactive processes increased the power of $\mathrm{G}_{4}$ in disrupting the charged membranes. The results are discussed in the context of general antibacterial actions as observed from other AMPs and membrane lytic actions. 


\section{Introduction}

Bacterial infections are becoming increasingly complicated to treat due to their ability to acquire resistance to the existing antimicrobial agents (1). Most antibacterial drugs work by either interfering with nucleic acid synthesis, inhibiting protein synthesis or altering metabolic pathways (2), but bacteria can often develop rapid resistance through mutation, selection or by acquiring genes from other bacteria $(3,4)$. Therefore, new drugs with different mechanisms of actions are needed to avoid the development of multiple resistances. One class of promising antimicrobial agents is the natural antimicrobial peptides (AMPs), which mainly work by disrupting bacterial membrane structures (5). Extensive studies have been undertaken to search for novel AMPs from various origins and sources, and develop biomimetic versions to evolve their membrane selective actions and targeting so that their therapeutic potential could be enhanced (6-8).

An important approach to aid the understanding of the mechanistic processes of membrane disruption is to study how AMPs interact with model membranes including spread lipid monolayers (9), vesicles $(10,11)$, and planar lipid bilayers (12-14). The exact choice of the model systems however depends on the experimental aims and the capacity to allow for rigorous control and manipulation of membrane composition including charge characteristics.

The binding selectivity of most AMPs to anionic lipid surfaces is of particular interest, as molecular interactions during this stage dictate the outcome of antibacterial treatments. The outer surfaces of bacterial cell membranes bear negative charges whilst those of the mammalian cell membranes largely remain neutral. This difference explains why cationic peptides are often preferred $(15,16)$. One class of antimicrobial peptides, composed of cationic, $\alpha$-helical peptides, has been particularly successful in offering antimicrobial properties (17). Most of them, being either natural, biomimetic, or rationally designed, have net positive charges and consist of more than $30 \%$ of hydrophobic residues $(18,19)$. However, rationally designed ones tend to be short and structurally well-defined, making it ideal for them to be used in mechanistic studies.

In this context, we have designed a new class of short cationic peptides with the general sequence $\mathrm{G}(\mathrm{IIKK})_{\mathrm{n}} \mathrm{I}-\mathrm{NH}_{2}$, with $n$ denoting the number of $\alpha$-helical repeats $(\mathrm{n}=1-4)(20)$. We have shown that peptides out of this series with $n=3-4$ can kill both gram positive and gram negative bacteria with high potency whilst maintaining low cytotoxicity against human erythrocytes cells, even at concentrations up to 10 -fold of minimal inhibitory concentrations (MICs). These peptides also 
have strong anticancer activities whilst remaining benign to mammalian cell hosts $(21,22)$. CD studies from membrane-mimetic small unilamellar vesicles showed that all the $\mathrm{G}(\mathrm{IIKK})_{2-4} \mathrm{I}-\mathrm{NH}_{2}$ peptides were unfolded in aqueous solution. They remained unstructured when exposed to the zwitterionic liposomes mimicking mammalian host cell membranes, but became transformed into $\alpha$-helix structures upon exposure to the negatively charged vesicles mimicking bacterial cell membranes. These actions follow the general features of many natural AMPs but the structural design enables us to learn how to tune up their antibacterial efficacy whilst minimizing their cytotoxicity against mammalian cell hosts. The structural studies via the binding and association of peptides onto model membranes can help provide useful insight at the molecular level about how AMPs interact with different lipid membranes.

In the work to be reported here, we control the binding of peptide G4 with the spread lipid monolayers on the surface of water using a Langmuir trough $(23,24)$, with the dynamic binding processes measured by Brewster angle microscopy (BAM) and neutron reflection (NR). Given that many literature studies have used single lipids as models and that this is the first study of the binding of $\mathrm{G}_{4}$ to lipid monolayers, we have chosen 1,2-dipalmitoyl-sn-glycero-3-phosphocholine (DPPC) and 1,2-dipalmitoyl-sn-glycero-3-phospho-(1'-rac-glycerol) (sodium salt) (DPPG) to approximate lipid membrane surfaces bearing different charge characteristics. A distinct advantage of NR is its structural sensitivity to deuterium labelling and both deuterated and hydrogenated versions of DPPC and DPPG were available for this work. The combined studies have allowed us to unravel structural details relating to the amount of the peptide bound and its location across the model lipid monolayers.

\section{Materials and methods}

\section{Materials}

The lipids were purchased from Avanti Polar Lipids (Alabaster, AL) and used as supplied. Stock solutions of fully hydrogenous and tail-deuterated $\left(\mathrm{d}_{62^{-}}\right)$1,2-dipalmitoyl-sn-glycero-3phosphocholine (DPPC) (synthetic purity > 99\%), 1,2-dipalmitoyl-sn-glycero-3-phospho-(1'-racglycerol) (sodium salt) (DPPG) (synthetic purity > 99\%) were prepared in chloroform/methanol (9:1) (Sigma-Aldrich, 99\%). The peptide, G(IIKK) $)_{4} \mathrm{I}-\mathrm{NH}_{2}$, (further denoted as $\mathrm{G}_{4}$ ) supplied by Shanghai Top-peptide Bio Co Ltd with 98\% purity, was synthesized using the standard solid phase Fmoc method and the synthesis and purification processes were previously described by Chen et 
al. (21). It was used without further purification. $\mathrm{G}_{4}$ is composed of 18 amino acids, and has a positive net charge of +9 (at neutral pH) (see Fig. S1 in the Supporting Information for its helical structure). The peptide solution was made using a phosphate buffer solution (PBS, $10 \mathrm{mM}, 137$ $\mathrm{mM} \mathrm{NaCl}, \mathrm{pH}=7.4$ ) using UHQ (Elgastat ultrapure water) grade water. The solution was diluted to the required concentration $(3 \mu \mathrm{M})$ by adding a concentrated peptide solution in the buffer subphases of the Langmuir trough. All other chemicals including $\mathrm{D}_{2} \mathrm{O}(99 \% \mathrm{D})$ were sourced from Sigma-Aldrich.

\section{Surface pressure measurements}

In all experiments the lipid monolayers were created at the air/water interface using a special Langmuir trough (Nima Technology Ltd, Coventry, UK) purposely designed to facilitate the neutron reflection measurement with due consideration of the sufficient beam footprint and liquid volume. Before starting the experiment the trough was thoroughly cleaned with chloroform, ethanol and UHQ grade water. Then the trough was filled with $80 \mathrm{ml}$ of PBS buffer and a certain amount of lipid stock solution was then carefully spread on the surface using a Hamilton syringe. After allowing about 10 minutes for the solvent to evaporate, the monolayer was compressed at a constant barrier speed of $5 \mathrm{~cm}^{2} / \mathrm{min}$. All the measurements were carried out at the room temperature of $22 \pm 2{ }^{\circ} \mathrm{C}$. The surface pressure-area curves $(\pi-\mathrm{A})$ of the lipids were recorded by compressing the barrier until reaching the minimum trough area. For the peptide binding experiments, before the peptide injection, the monolayer was compressed and held at the initial surface pressure of $15 \mathrm{mN} / \mathrm{m}$. The surface area was then fixed by parking the trough barrier. The peptide solution was then slowly injected in the subphase underneath the lipid layer via a syringe with a long bent needle introduced from the other side of the barrier (Fig. S2) and was dispersed in the entire trough to encourage uniform distribution of the peptide solution. Then the surface pressure-time curves were recorded. All the experiments were repeated at least three times, to ensure the reproducibility of the measurements.

\section{Brewster angle microscopy}

Brewster angle microscopy (BAM) measurements were recorded using an $\mathrm{EP}^{3}$ imaging ellipsometer (Nanofilm Technology, Goettingen, Germany). It was mounted on top of the Langmuir trough. This setup allowed for a simultaneous measurement of surface pressure and BAM imaging of the in-plane film structures and all measurements were repeated twice. The 
microscope was equipped with a frequency doubled $\mathrm{Nd}$ :YaG laser $(532 \mathrm{~nm}, 20 \mathrm{~mW})$, polarizer, analyser and CCD camera. The images were taken using a $10 \times$ objective lens. The reflectivity of the p-polarised light at the air/water interface at the Brewster angle $\left(53.15^{\circ}\right)$ is nil; hence any changes in the refractive index profile across the interface result in the increased reflection. BAM imaging has been widely used in studying the morphological features of lipid monolayers at different surface pressures (25), as well as in the binding process from peptide/protein $(26,27)$. Upon the formation of the lipid monolayer, the barrier was compressed to the surface pressure of $15 \mathrm{mN} / \mathrm{m}$ and, once the monolayer was stable, BAM images were recorded for the lipids only before peptide addition. Afterwards, the surface area was held constant. The peptide solution was then injected in the subphase, followed by BAM image recording at regular intervals for 150 minutes whilst the surface pressure was monitored. All BAM images were background subtracted, and then corrected and processed using the imageJ software.

\section{Neutron reflectivity}

The NR measurements were carried out using the FIGARO reflectometer at the Institut LaueLangevin (Grenoble, France) (28). The time-of-flight instrument was used with a chopper pair which gave the neutron wavelength range between 2 and $30 \AA$ and a $d \lambda / \lambda$ of $7.0 \%$. The data were acquired at two incident angles of $0.62^{\circ}$ and $3.8^{\circ}$, giving a momentum transfer range $\left(Q_{z}\right)$ from about 0.005 to $0.4 \AA^{-1}$. The instrument was calibrated using a clean $\mathrm{D}_{2} \mathrm{O}$ subphase. The background was subtracted using the $2 \mathrm{D}$ detector.

Variation in the scattering length density (SLD) along the surface normal direction determines the specular neutron reflectivity. Because the scattering lengths for hydrogen and deuterium are different, isotopic substitutions can give different reflectivity profiles for a given interfacial structure. Neutron reflectivity profiles were measured using lipids with deuterated acyl chains and a mixture of deuterated and hydrogenated lipids to give the SLD of the tails matched to air or NRW (null reflective water). This allowed a precise determination of the interfacial adsorbed amount and the thickness of the acyl chain region. Peptide binding to DPPC and DPPG monolayers was measured after the NR profiles for the lipid monolayers were recorded. Following injection of the peptide in the subphase, the reflectivity was updated every $4 \mathrm{~min}$, which allowed us to conduct a dynamic analysis of the peptide-lipid interaction. 
The raw NR data were analysed by two different types of data treatment. First, a low-Q analysis was deployed to follow the compositional changes over time. In this approach, the low-Q data were reduced over a restricted Q-range of $0.01-0.03 \AA^{-1}$ to minimize the influence of details of the structural model on the fitted lipid surface concentrations or peptide binding $(31,32)$. The samples used were cm-DPPC, d-DPPC, and cm-DPPG and d-DPPG, respectively, measured in NRW, before and after injecting the $\mathrm{G}_{4}$ peptide, where the term ' $\mathrm{cm}$ ' refers to the lipid chains that were 'contrast matched' to give zero SLD. Second, a full Q-range analysis was carried out where the data recorded at different angles were combined. The reflectivity profiles for each data set were then simultaneously fitted using Motofit (29) which uses an optical matrix formalism (30) to fit Abeles layer models to the interfacial structure.

In the case of the lipid-peptide system, a single layer model in the low-Q range involving SLD $(\rho)$ and thickness $(\tau)$, was used to solve the following simultaneous equations:

$$
\begin{aligned}
& (\rho \tau)_{1}=N_{A}\left(\Gamma_{c m-l i p i d} b_{c m-l i p i d}+\Gamma_{\text {peptide }} b_{\text {peptide }}\right) \\
& (\rho \tau)_{2}=N_{A}\left(\Gamma_{d \text {-lipid }} b_{d-\text {-lipid }}+\Gamma_{\text {peptide }} b_{\text {peptide }}\right)
\end{aligned}
$$

where $N_{A}$ is the Avogadro's number, $\Gamma$ is the surface concentration (measured in $\mathrm{mol} / \mathrm{m}^{2}$ ), which for 'cm' and 'd' lipids has the same values, and $b$ is the respective calculated scattering lengths. The benefit of this approach is its insensitivity to any structural detail, as a single layer model was sufficient to extract both the lipid surface coverage and peptide binding.

For the structural analysis of the interfacial layer, the data from the different isotopic contrasts were fitted together. For the lipid only systems, a two-layer model fit was appropriate, with the top layer containing lipid tails and bottom layer containing lipid head groups. The tail volume fraction was fixed to unity for simplicity, with the hydrated head layer being fitted taking into account the volume fraction of the solvent as well, and the summation of the head volume fraction $\left(\varphi_{\text {head }}\right)$ and solvent volume fraction $\left(\varphi_{\text {solvent }}\right)$ being equal to unity. To ensure the same number of the tails and heads we used the following equations:

$$
\begin{aligned}
\Gamma_{\text {tails }} & =\frac{\rho_{\text {tails }} \tau_{\text {tails }}}{\sum b_{\text {tail }} N_{A}} \\
\Gamma_{\text {head }} & =\frac{\rho_{\text {head }} \tau_{\text {head }} \varphi_{\text {head }}}{\sum b_{\text {head }} N_{A}}
\end{aligned}
$$




$$
\Gamma_{\text {tails }}=\Gamma_{\text {head }}
$$

Upon addition of peptide, however, peptide may become associated into both head and acyl chain region and alter the interfacial packing resulting in different interfacial composition. To take into account the peptide amount into each of the layers, the volume fraction of the peptide $\left(\varphi_{\text {peptide }}\right)$ needs to be considered in Eq. 3 and Eq. 4 by following Eq. 6:

$$
\rho_{\text {fit }}=\rho_{\text {lipid }} \varphi_{\text {lipid }}+\rho_{\text {peptide }} \varphi_{\text {peptide }}+\rho_{\text {solvent }} \varphi_{\text {solvent }}
$$

where $\varphi_{\text {lipid }}$ is the lipid volume fraction (heads or tails), $\rho_{\text {lipid }}$ represents the calculated values of the SLD of the lipid molecules (heads or tails), $\rho_{\text {peptide }}$ the calculated SLD of the $\mathrm{G}_{4}$ peptide and $\rho_{\text {fit }}$ is the fitted SLD value of the layer concerned. The surface concentration for each component was then determined by using Eq. 3-5 and taking into account the volume fractions of lipid, pepide and water where it is assumed that the tail layer contains no water and that the space filling condition holds for the head layer with $\varphi_{\text {lipid }}+\varphi_{\text {peptide }}+\varphi_{\text {solvent }}=1$. The area per molecule (A) of each species can be calculated using:

$$
A=\frac{1}{\Gamma N_{A}}
$$

The SLD values for the lipid and peptide components used in the NR data analysis are summarized in Table S1 in the Supporting Information. The molecular volume and SLD values of the peptide were calculated using Biomolecular SLD Calculator developed by ISIS Neutron Facility (http://psldc.isis.rl.ac.uk/Psldc/).

\section{Results and Discussion}

\section{Surface pressure measurements}

The surface pressure-area $(\pi-\mathrm{A})$ isotherms recorded for DPPC and DPPG monolayers on PBS buffer subphase are shown in Fig.1a. Both DPPC and DPPG monolayers show similar phase transition behaviour, but the exact $\pi$-A curves differ, showing the transitions occurred at slightly different area per molecule values associated with the different head groups and isotopic substitutions. These changes arising from deuterium labelling and lipid head group caused slightly different molecular interactions and the results as shown in Fig. 1a were consistent with the reported studies (9). 
The peptide binding experiments were carried out at the initial surface pressure of $15 \mathrm{mN} / \mathrm{m}$. Even though this surface pressure is not an exact mimetic of that found in the cell membranes, but it is the optimal pressure to obtain both stable monolayers and noticeable differences to enable comparative studies for the two lipid systems. At this pressure the lipids are in a so-called tilted condensed phase, with the area per molecule of $53 \pm 2 \AA^{2}$ for the DPPC monolayer and $54 \pm 2 \AA^{2}$ for the DPPG monolayer, respectively. Fig. 1b shows the surface pressure changes against time over the first $150 \mathrm{~min}$ after peptide injection into the subphase, with the inset showing the zoomed plot for the first $10 \mathrm{~min}$ of surface pressure changes. The final peptide concentration in the subphase was fixed at $3 \mu \mathrm{M}$. This concentration was close to the minimum inhibitory concentrations of the $\mathrm{G}_{4}$ peptide against bacterial growth and the $50 \%$ inhibition concentrations against cancerous growth (which are around 0.5-10 $\mu \mathrm{M}$ ) as previously described by $\mathrm{Hu}$ el al. (20). It should be noted that measurements were also made at the high surface pressure of $28 \mathrm{mN} / \mathrm{m}$. Similar trend of surface tension reduction upon peptide binding was observed with the extent of surface pressure rising being reduced. The difference should not affect the main structural and morphological features from BAM and NR studies.

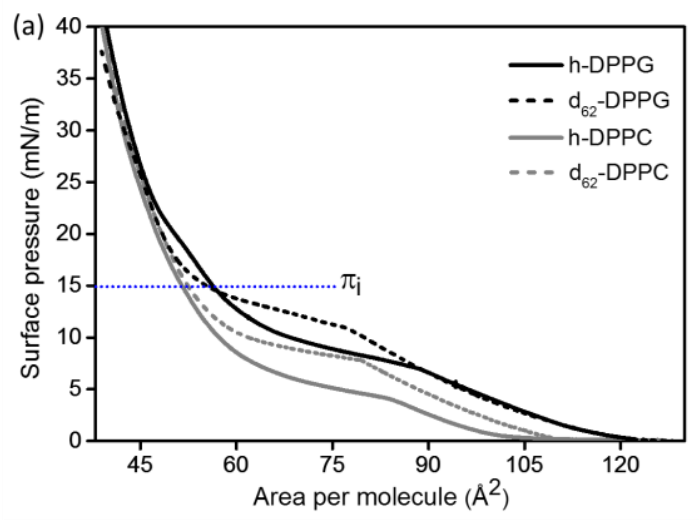

Figure 1. Surface pressure plots in (a) showing the $\pi$ A curves for hydrogenous and tail-deutarated DPPC and DPPG, respectively, spread on PBS buffer subphase, with $\pi_{i}$ indicating the initial pressure of the lipids at which the peptide was injected for all the binding experiments, and in (b) showing the changes in $\pi$ versus time after injection of the $G_{4}$ peptide under DPPC and DPPG monolayers at intial pressures of 15 $\mathrm{mN} / \mathrm{m}$, with the inset showing the zoomed surface pressure changes for the first $10 \mathrm{~min}$. 


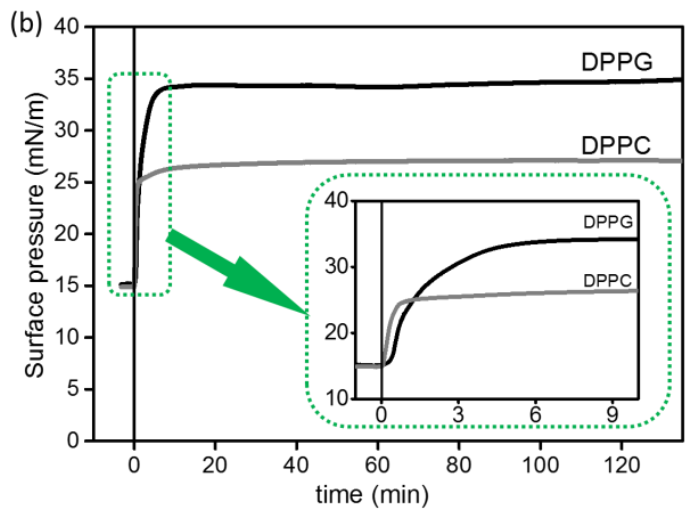

Once the peptide was injected into the subphase below the lipid monolayers, the surface pressure rose sharply within the first 5-6 min, followed by a slow adjustment over the next 10 min before it tended to a plateau in each case. In the case of the DPPC monolayer, the final surface pressure increase $\left(\Delta \pi=\pi_{\max }-\pi_{i}\right)$ was $10 \pm 2 \mathrm{mN} / \mathrm{m}$. The pressure rise could arise from peptide accumulation below the lipid layer, interaction with the zwitterionic headgroups, insertion into the hydrocarbon tail region through hydrophobic interactions or a combination of these events. In the case of the DPPG monolayer, however, the surface pressure increase almost doubled, reaching a value of $21 \pm 3 \mathrm{mN} / \mathrm{m}$. The difference is attributed to the strong electrostatic interaction between the anionic lipid head group and the cationic peptide, followed by hydrophobic interaction associated with the insertion into the DPPG monolayer. Whilst it remains difficult to infer the structural implications from the surface pressure alone, these measurements help define the systems for BAM and NR studies.

Lipid monolayers can have binary or more components to mimic the composition and physiology of the membrane leaflets concerned. Whilst more components can make better approximations, complexity also arises from the interactions between components. Incorporation of unsaturation and charges can also alter phase transition $(33,34)$, but mixing of peptide into these lipid monolayers all leads to the rise of surface pressure, though the exact amount of the changes may vary. By using single lipid components and well defined measurement conditions in this work, the main structural features arising from charge interaction could be unravelled unambiguously.

\section{Morphological imaging from Brewster angle microscopy (BAM)}


The BAM images of DPPC and DPPG monolayers were taken before and after the $\mathrm{G}_{4}$ peptide injection to examine whether the peptide would induce any measurable morphological changes in the in-plane structure of the monolayers, with representative images shown in Fig. 2. Prior to peptide addition, the images from the DPPC and DPPG monolayers were taken with $\pi_{i}$ being fixed at $15 \mathrm{mN} / \mathrm{m}$ (time $\mathrm{t}=0$ in Fig. 2). Both lipid monolayers showed well-defined lateral anisotropic domain structures, with distinct features arising from the segregated lipid domains containing molecules of different tilt angles. The DPPC monolayer forms irregular, counter-clockwise lobeshape domains, caused by the chirality of the DPPC molecules, whereas for DPPG the liquid condensed phase forms polygonal shaped domains. $(35,24)$.
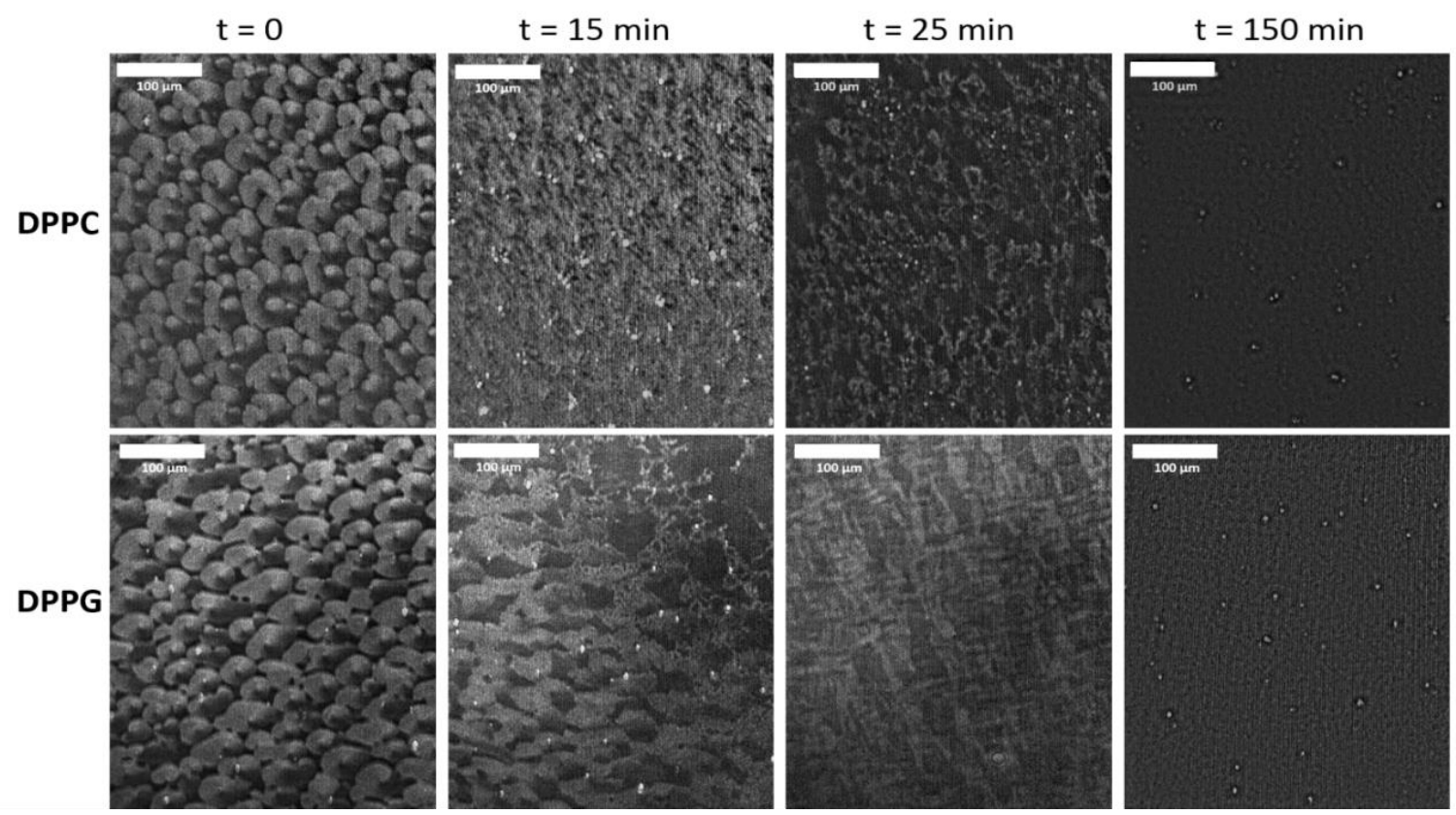

Figure 2. Brewster angle microscopy images recorded before $(t=0)$ and after $G_{4}$ peptide injection to the aqueous subphase underneath tilted condensed phase DPPC (top) and DPPG (bottom) monolayers. The progressive changes indicate the binding and association of the $G_{4}$ peptide with each monolayer. The initial surface pressures were $15 \mathrm{mN} / \mathrm{m}$.

Binding of peptide molecules resulted in noticeable changes in the structure of the lateral domains, and there was a clear time-dependent process for each lipid monolayer. Approximately $15 \mathrm{~min}$ after peptide injection, there was a striking transformation in the morphology of both monolayers. Note that the BAM images were recorded simultaneously with the surface pressure recording. Together they showed that the drastic changes in the lateral structures of the lipid films 
corresponded well with the time for the peptide binding to reach the maximum pressure increase and that the processes subsequently moved towards the plateau region in each case. Once the peptide was bound to the layers, it had a fluidizing effect on the ordered lipid domains. Similar effects have been reported previously for other peptide-lipid monolayer systems (36, 37). The inhomogeneous features associated with domains or fragments in the monolayers started to disappear, and these changes coincided with the plateauing of the limiting surface pressure for each system. The fluidization, which took place almost after $60 \mathrm{~min}$, was broadly similar for both lipid monolayers in spite of different lipid head groups. The surface morphology was then characterized by a predominantly homogeneous in-plane structure dotted with small and illdefined domains. Some of these small domains could be observed even after 3 hours, the longest period of image recording. Thus, for both zwitterionic and anionic lipid monolayers, the dynamic peptide binding led to similar domain structure transition processes and the final peptide associated layers all resulted in the predominantly homogenous surface within the micrometre resolution of the measurements. These images demonstrate the ability of the peptide to interact and influence the lipid lateral organization by a fluidizing effect. Even though there was a large difference in the final surface pressure from the two monolayer models, the visual BAM images revealed a broadly similar surface morphological transitional process upon peptide binding.

\section{Structural studies by neutron reflection}

Lipid monolayer structures NR measurements were first made to determine the structures of the DPPC and DPPG lipid monolayers alone. For each lipid, the reflectivity profiles were recorded in 4 isotopic contrasts: cm-lipid on NRW, d-lipid on NRW, cm-lipid on $\mathrm{D}_{2} \mathrm{O}$ and d-lipid on $\mathrm{D}_{2} \mathrm{O}$, all at a fixed pressure of $15 \mathrm{mN} / \mathrm{m}$. These reflectivity profiles were fitted by adopting a two-layer model, as it represented the simplest structure that fitted all the measured data. In this model, the first layer contains all acyl chains and the second contains all head groups with remaining space filled by water. Fig. 3 shows the model fits to the measured data with the background being subtracted (but still containing a residual background of $7 \times 10^{-7}$ ). Fig. 3a depicts the measured NR profiles and the best fits for the DPPC monolayers, with the SLD profiles across the interface shown in the inset; Fig. 3 b depicts the corresponding data and fits for the DPPG monolayers. The structural parameters obtained from the fits are listed in Table S2. The thicknesses of the acyl chains for both DPPC and DPPG were found to be $15.5 \AA$. The fitting at this surface pressure led to a volume fraction of $0.48 \pm 0.05$ for the DPPC head group, whereas for DPPG each head group 
was slightly more densely packed, with a volume fraction of $0.55 \pm 0.03$. The area per lipid molecule was found to be comparable with those determined from the Langmuir trough experiments, with $53 \pm 2 \AA^{2}$ for both DPPC and DPPG. From the SLD values and the fitted thicknesses from the $\mathrm{D}_{2} \mathrm{O}$ contrasts, we estimated that each DPPC head was associated with 11 water molecules and each DPPG had 8 water molecules associated.
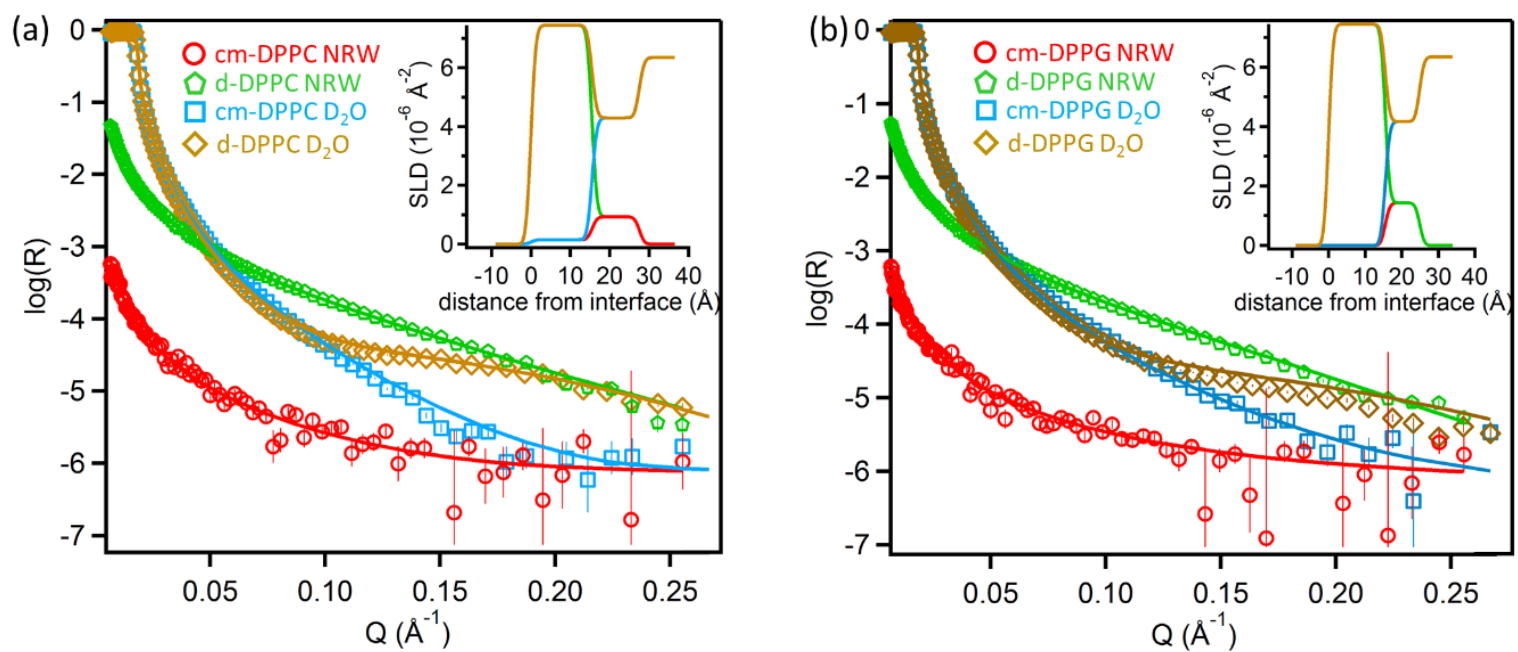

Figure 3. NR profiles for (a) DPPC and (b) DPPG monolayers at $15 \mathrm{mN} / \mathrm{m}$ involving contrast-matched lipids (cm-DPPC, cm-DPPG) on NRW (O), chain-deuterated lipids (d-DPPC, d-DPPG) on NRW (口), contrast-matched lipids on $\mathrm{D}_{2} \mathrm{O}(\square)$ and chain-deuterated lipids on $\mathrm{D}_{2} \mathrm{O}(\diamond)$. The simultaneous two-layer fits are shown as solid lines (-) with matching colours for each contrast. The SLD profiles obtained from the fits as a function of distance along the interface normal are plotted with the corresponding colours for each contrast.

Dynamic process of peptide binding The surface tension measurements have indicated a fast initial dynamic step of binding or co-adsorption of the $\mathrm{G}_{4}$ peptide onto lipid monolayers over the first 2-5 min, followed by slow transition and relaxation steps. In contrast, the signal of NR relies on statistical counting from the interface. It was found that by measuring the binding of the G4 peptide onto either 'cm' or 'd' lipid monolayers in NRW, each run could be taken in 4 min, but the reflectivity must be recorded over the high flux range at the low incidence angle of the neutron beam (32). This was achieved by undertaking the reflectivity measurements over the low-Q range. In order to calculate the adsorbed amount, we have applied Eq. 1 and Eq. 2 to the two contrasts mentioned above, i.e 'cm' and 'd' lipid on NRW. Fig S3 shows the set of reflectivity profiles measured upon peptide binding onto DPPC and DPPG monolayers at different time intervals, with 
Fig. S4 showing the amount of lipid remaining and amount of peptide bound as calculated from the reflectivity profiles. The text given in the Support Information provides more detailed information about the calculation from the reflectivity profiles shown in Figure S3.

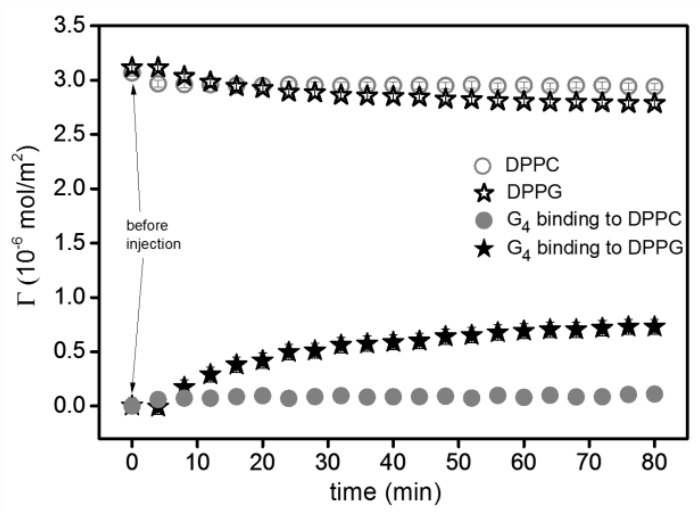

Figure 4. Surface concentration values as a function of time representing simultaneous peptide binding to the interface of DPPC and DPPG monolayers together with the lipid loss.

Fig. 4 shows the surface concentration of the peptide upon injection under lipid monolayers plotted against time. Also plotted are the changes of the total lipid surface concentration with time, showing a clear decreasing trend with the simultaneous peptide adsorption. The time zero is considered as being the surface coverage values for the lipids only. Although the plateau was reached within the first 5-8 minutes after the peptide injection (Fig. 1b), the NR data clearly shows that the equilibrium is much slower, being reached after $50-80 \mathrm{~min}$. This is clearly the case for the binding of the peptide to DPPG, but is unclear for DPPC due to the very low level of peptide binding. Note that before peptide injection, the initial surface concentrations for DPPC and DPPG monolayers were similar, $3.06 \mu \mathrm{mol} / \mathrm{m}^{2}$ for DPPC and $3.12 \mu \mathrm{mol} / \mathrm{m}^{2}$ for DPPG. The dynamic peptide binding to the zwitterionic DPPC monolayer over a period of 80 min remains very low, and the final surface concentration of the lipids was $2.93 \mu \mathrm{mol} / \mathrm{m}^{2}$ with a total peptide adsorbed amount of $0.13 \mu \mathrm{mol} / \mathrm{m}^{2}$. In the case of peptide-DPPG system, however, there was a clear timedependent increase of the total peptide binding, and the simultaneous loss of the lipid was more pronounced. After $80 \mathrm{~min}$, the system tended to equilibration. The peptide adsorbed amount reached $0.73 \mu \mathrm{mol} / \mathrm{m}^{2}$ whereas that for the anionic DPPG monolayer decreased to $2.79 \mu \mathrm{mol} / \mathrm{m}^{2}$. 
Structural analysis of $G_{4}$ bound to DPPC and DPPG interfacial layers The structures of the DPPC and DPPG interfacial layers after $\mathrm{G}_{4}$ peptide binding reached equilibration were measured by recording the NR profiles over the entire Q-range using 4 different isotopic contrasts as detailed in the Experimental Section. On the basis of the two-layer model representation for the lipid only interface (Fig. 3), we first attempted to fit the data in all contrasts for the lipid monolayers incorporating the $\mathrm{G}_{4}$ peptide. The best fits are plotted in Fig. S5 and Fig. S6 and the fitted parameters are listed in Table S3 and Table S4. In this model, it was assumed that the peptide would largely be associated with the head group layer, but the fits did not give satisfactory results, as the head group containing layer (the $2^{\text {nd }}$ layer) became unrealistically thick. This together with the occurrence of the mismatching fringes in the NR profiles fitted by these values cannot support this model.
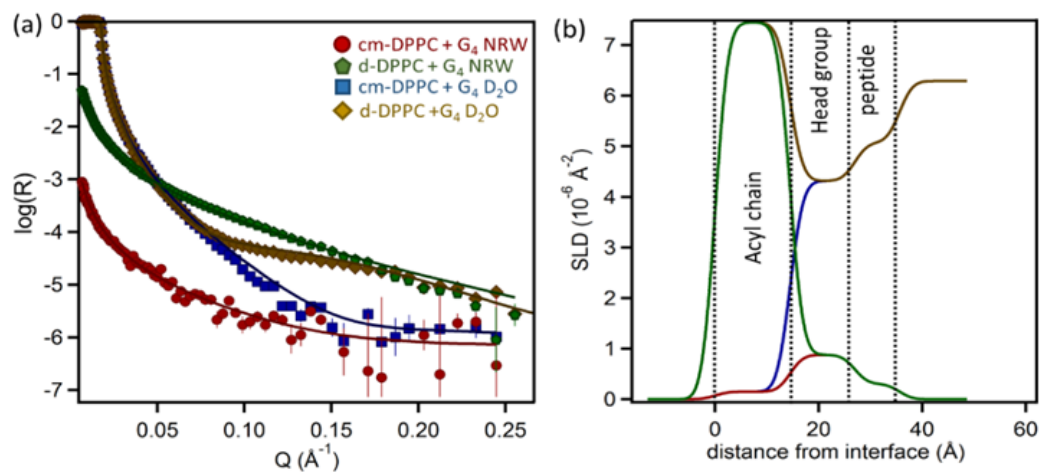

Figure 5 NR profiles for a best threelayer model fit to the data of an equilibrium $G_{4}$ adsorbed to (a) DPPC and (c) DPPG monolayers for contrastmatched lipids (cm-DPPC, cm-DPPG) on NRW $(-)$, chain-deuterated lipid ( $d$ DPPC, d-DPPG) on NRW ( ), contrast-matched lipid on $\mathrm{D}_{2} \mathrm{O}$ (口) and
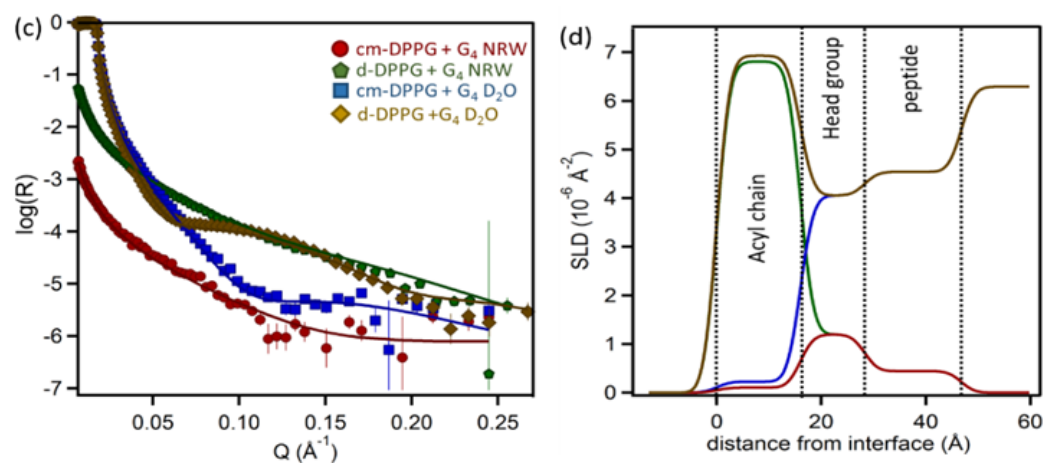
chain-deuterated lipid on $\mathrm{D}_{2} \mathrm{O}($ ). The simultaneous three-layer fits are shown as solid lines (-) with corresponding colours for each contrast. The SLD profiles, obtained are represented for the equilibrium $G_{4}$ adsorbed (b) DPPC and (d) DPPG monolayers from the four contrasts, with the corresponding colours for each contrast.

We subsequently deployed a three-layer model fit for each lipid-peptide system to achieve physically more meaningful fits. The model consisted of acyl chains with or without peptide in the top $1^{\text {st }}$ layer, lipid head groups with peptide in the $2^{\text {nd }}$ layer and peptide only in the $3^{\text {rd }}$ layer with the remaining spaces in the $2^{\text {nd }}$ and $3^{\text {rd }}$ layers filled by water. Similar models have been used to account for the binding of proteins onto lipid monolayers (24). Fig. 5 shows the reflectivity profiles and the best three-layer fits to the DPPC (Fig. 5a) and DPPG (Fig. 5c) monolayers 
incorporating the $\mathrm{G}_{4}$ peptide and the SLD profiles obtained from the respective fits are shown in Fig. $5 \mathrm{~b}$ and $5 \mathrm{~d}$. The structural parameters obtained from the respective fits are listed in Table 1. The detailed fitting parameters for all contrasts are summarized in Tables S5 and S6. As shown in Fig. 5a and 5b, peptide binding to the zwitterionic DPPC monolayer did not induce significant structural changes across the interfacial layer, with the top acyl layer remaining constant at $14.5 \AA$ without any sign of peptide uptake, but peptide molecules were mainly associated with the head group layer with its thickness extended to $12.5 \AA$. In addition, the extended peptide-only layer was fitted to $8.5 \AA$. The total amount of the peptide was only at $0.17 \mu \mathrm{mol} / \mathrm{m}^{2}$, with $14 \%$ being adsorbed to the head group region $\left(0.02 \mu \mathrm{mol} / \mathrm{m}^{2}\right)$, and $86 \%$ underneath the head group region $\left(0.15 \mu \mathrm{mol} / \mathrm{m}^{2}\right)$. The full Q-range data analysis also allowed us to calculate the amount of the lipid within the monolayer, consistent with the outcome of slight reduction of the DPPC content upon peptide binding as calculated from the low-Q range data analysis.

Table 1. Parameters obtained from the best three-layer model fits to the DPPC and DPPG monolayers with $G_{4}$ bound at equilibrium after an initial surface pressure of $15 \mathrm{mN} / \mathrm{m}$. The errors quoted denote the range of variations beyond which deviations in reflectivity fitting became noticeable.

\begin{tabular}{clllllll}
\hline Layer & $\tau(\AA)$ & $\varphi_{\text {lipid }}$ & $\varphi_{\text {peptide }}$ & $\varphi_{\text {solvent }}$ & $\begin{array}{c}\mathrm{A}_{\text {lipid end }} \\
\left(\AA^{2}\right)\end{array}$ & $\begin{array}{c}\Gamma_{\text {lipid end }}\left(10^{-6}\right. \\
\left.\mathrm{mol} / \mathrm{m}^{2}\right)\end{array}$ & $\begin{array}{c}\Gamma_{\text {peptide }}\left(10^{-6}\right. \\
\left.\mathrm{mol} / \mathrm{m}^{2}\right)\end{array}$ \\
\hline DPPC & & & & & & \\
\hline$\left(1^{\text {st }}\right)$ acyl chain & $14.5 \pm 1$ & $0.95 \pm 0.05$ & $\mathrm{~N} / \mathrm{A}$ & $\mathrm{N} / \mathrm{A}$ & $56.5 \pm 2$ & $2.93 \pm 0.02$ & $\mathrm{~N} / \mathrm{A}$ \\
$\left(2^{\text {nd }}\right)$ head group & $12.5 \pm 1$ & $0.43 \pm 0.05$ & $0.03 \pm 0.003$ & $0.54 \pm 0.03$ & $56.5 \pm 2$ & $2.93 \pm 0.02$ & $0.02 \pm 0.002$ \\
$\left(3^{\text {rd }}\right)$ peptide & $8.5 \pm 1$ & N/A & $0.7 \pm 0.07$ & $0.3 \pm 0.03$ & N/A & N/A & $0.15 \pm 0.02$ \\
\hline
\end{tabular}




\begin{tabular}{|c|c|c|c|c|c|c|}
\hline DPPG & & & & & & \\
\hline$\left(1^{\text {st }}\right)$ acyl chain & $16 \pm 1 \quad 0.9 \pm 0.05$ & $0.1 \pm 0.01$ & $\mathrm{~N} / \mathrm{A}$ & $57 \pm 2$ & $2.9 \pm 0.02$ & $0.09 \pm 0.01$ \\
\hline$\left(2^{\text {nd }}\right)$ head group & $12.5 \pm 1 \quad 0.4 \pm 0.03$ & $0.18 \pm 0.02$ & $0.42 \pm 0.03$ & $57 \pm 2$ & $2.9 \pm 0.02$ & $0.12 \pm 0.01$ \\
\hline$\left(3^{\text {rd }}\right)$ peptide & $18.5 \pm 2 \mathrm{~N} / \mathrm{A}$ & $0.44 \pm 0.04$ & $0.56 \pm 0.05$ & $\mathrm{~N} / \mathrm{A}$ & N/A & $0.46 \pm 0.04$ \\
\hline
\end{tabular}

In contrast, the amount of peptide $\mathrm{G}_{4}$ adsorbed to the DPPG monolayer was much more, as evident from the NR and SLD profiles shown in Fig. 5c and 5d. The total peptide amount was calculated to be $0.67 \mu \mathrm{mol} / \mathrm{m}^{2}$, with $13 \%$ distributed into the acyl chain region $\left(0.09 \mu \mathrm{mol} / \mathrm{m}^{2}\right), 18 \%$ into the head group region $\left(0.12 \mu \mathrm{mol} / \mathrm{m}^{2}\right)$ and $68 \%\left(0.46 \mu \mathrm{mol} / \mathrm{m}^{2}\right)$ in the peptide-only region. The total peptide bound was about 4 times greater than that found from the DPPC monolayer, consistent with the outcome from the low-Q range data analysis. Furthermore, the peptide was more aggressive at removing the DPPG lipid molecules from the interface: as much as $10 \%$ of DPPG disappeared during the adsorption process, in contrast to only $2-3 \%$ of lipid removal upon binding to the DPPC monolayer. The stronger strength of the $\mathrm{G}_{4}$ peptide binding to the DPPG monolayer was mainly demonstrated in the increased penetration across the entire lipid regions, manifested by its stronger affinity to the anionic lipids due to the electrostatic interaction. Peptide penetration into the acyl tail region also indicated the synergistic effect from the hydrophobic interaction to go across cell membranes, a common feature of AMPs during the early stage of membrane disruptions.

Extensive cell work has focused on assessing the efficacy and toxicity of natural and designed AMPs using cell and animal models (1-9,11,21,22). AMPs target bacterial membranes and kill bacteria by causing structural disruptions. In contrast, most antibiotics work by disrupting enzymatic pathways. Because it is far more difficult to develop antimicrobial resistance (AMR) against the non-specific membrane targeting, AMPs hold great promise in fighting against AMR, which is fast evolving into the possible global healthcare threat. With many AMPs coming into the horizon of high potency, more and more studies are being devoted to the understanding of their toxicity. In addition to the measurement of the haemolysis of human red blood cells (hRBCs), recent research has also started to screen the toxicity of AMPs to other primary human cells such as fibroblasts via MTT assays, thereby widening the assessment of the selective responses of AMPs from both sides. 
Although the BAM imaging only revealed morphological features of the lipid domains with the micron resolution, these morphological changes informed the impact of peptide interaction with the model monolayers. The main observations were that morphological changes from both systems were similar and that the dynamic processes were much slower than the surface tension changes. The NR measurements revealed that whilst the amount of the peptide bound to the DPPC monolayer was small the binding was faster. In contrast, the amount of the peptide bound to the DPPG monolayer occurred much more slowly and the majority of the peptide binding occurred over the first 30-40 min, a dynamic process that would match the surface morphological changes as observed from the BAM imaging. It can thus be concluded that although all three techniques deployed in this study revealed a common feature of dynamic changes upon peptide binding, the exact times for changes in surface tension, surface lipid morphologies and the amount of peptide bound are quite different.

The resolution of NR measurements is limited by the availability of deuterated samples. As chain deuterated DPPC and DPPG are readily available, these two lipids have been selected as the model monolayer surfaces. The combined NR measurements under different isotopic contrasts confirmed not only the greater extent of $\mathrm{G}_{4}$ peptide binding into the DPPG lipid monolayer but also the more destructive interactions evident from the greater extent of loss of DPPG lipids from the interface and the more extensive penetration of the peptide. These differences are well linked to the selective responses of these peptides in their antimicrobial actions $(18,20,22)$. At the molecular level, the differences are well correlated to the electrostatic interaction between the cationic peptide and the anionic head groups of DPPG lipids that facilitates the preferential peptide binding and subsequent insertion.

The different peptide binding to the two types of lipid monolayers and the impact on the interfacial structures are outlined in Fig. 6. These schematic representations are also supported by the volume fraction distributions of the lipid tail and head regions with and without the binding from $\mathrm{G}_{4}$ peptide. The drawings have taken into account the higher level of peptide binding to the anionic lipid monolayer and the less extent of peptide binding to the zwitterionic lipid monolayer, consistent with the selective antibacterial actions observed in co-culturing experiments $(18,20)$. The cartoon in Fig. 6a depicts the low peptide association surrounding the head group region of DPPC molecules where the peptide molecules lay flat or tilted. These molecular conformations fit to the fitted head group region, showing that the peptides predominantly adopt a flat-on orientation 
in parallel with the interface. In contrast, more peptide molecules are bound to the DPPG monolayer. Fig. $6 \mathrm{~b}$ depicts this feature with a clear emphasis of the penetration of the peptide into the acyl chain region as well. Furthermore, the peptide molecules must predominantly adopt the $\alpha$ helical structuring triggered by the hydrophobic interaction with the acyl chain region with a large extended region into the bulk solution. Transformation from non-ordered to $\alpha$-helical structuring following electrostatic and hydrophobic interactions has been well demonstrated from lipid vesicles $(20,22,40)$.
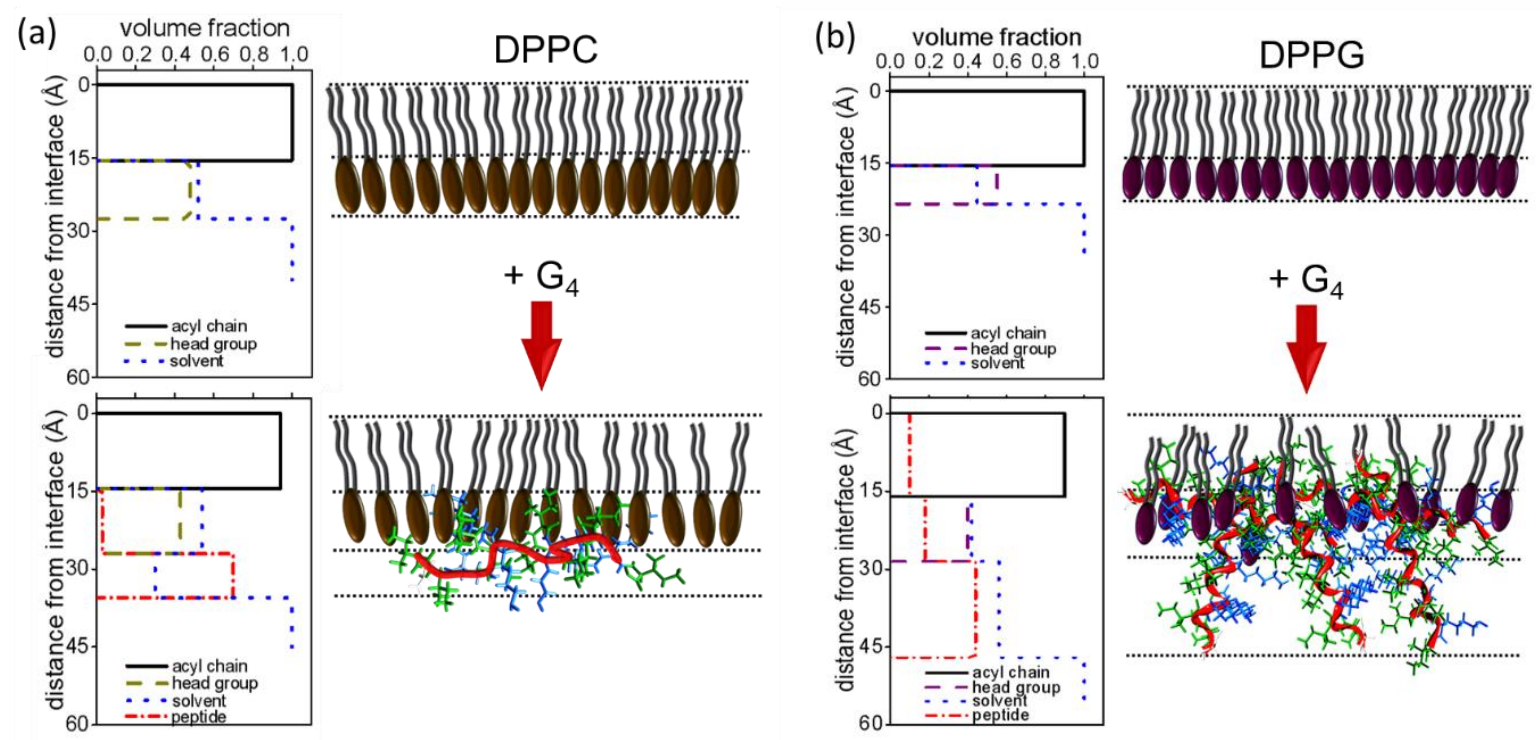

Figure 6. Volume fraction profiles as a function of distance along the interface normal and cartoon representation of the interfacial structure of the condensed phase DPPC (a) and DPPG (b) lipids before and after peptide addition. The Lys residues are coloured in blue, and the Ile in green.

Membrane permeation and formation of barrel-like nanoaggregates are important to aid membrane lytic actions once the peptides have selectively recognized and became associated with the negatively charged membranes. Although our own study did not extend to a determination of the in-plane formation of peptide aggregates, other studies by neutron scattering from lipid multilayers incorporating AMPs such as melittin and LL-37 have elucidated their existence in the lipid membranes $(38,39)$. This work has added to the ongoing studies by illustrating the impact arising from the charge difference of the membrane surfaces and subsequent structural details associated with the lipid losses and structural disruptions. These molecular structural differences upon peptide binding are important towards the selective actions between antibacterial efficacy and 
mildness to mammalian host cells. Neville et al (41) also demonstrated selective binding of LL-37 peptides onto lipid monolayers with and without charges by synchrotron radiation. Whilst the NR data follows the same trend, it is capable of distinguishing lipids from peptides and was thus able to determine the amount and relative location of the two components even if the amount of the $\mathrm{G}_{4}$ binding was low.

As this is the first study of $\mathrm{G}_{4}$ peptide binding, we have focused on the measurements of single lipid components for the best structural resolution from NR. Experiments reporting the use of 2 or more lipid component monolayers and other AMPs have given similar observations in terms of surface pressure rising $(9,24,33,34)$ and basic binding mechanism. Knyght et al $(34)$ have studied the binding of Rhesus $\theta$-defensin (RTD-1) and Porcine Protegrin-1 (PG-1) with binary component anionic lipid monolayers by NR aided with computer modelling. Their NR results also confirmed the extensive mixing of these AMPs with the charged lipid monolayers, but the more amphiphilic PG-1 becoming fully mixed with the lipid monolayer. In contrast, the defensin RTD-1 is less amphiphilically balanced and was observed to insert less deeply. As natural peptides, both RTD-1 and PG-1 have the same 18 amino acids as $\mathrm{G}_{4}$, but they are structurally more complex, making it more difficult to link molecular structures to their membrane disruptions.

\section{Conclusion}

Lipid monolayer models have enabled us to facilitate the direct measurements of peptide binding to lipids at a fluid interface using surface tension, BAM and NR under similar experimental conditions. The results presented here have illustrated different interactions between antimicrobial $\mathrm{G}_{4}$ peptide and model phospholipid monolayers mimicking membranes with different charge characteristics. Our studies revealed that the binding processes occurred within minutes after peptide injection and that the peptide displayed a strong preference to the charged membrane over the zwitterionic surface, an observation entirely consistent with their antibacterial activity and low toxicity. Whilst the trend of the dynamic changes was similar, increase in surface tension upon peptide binding happened much faster than the morphological changes of the lipid films and the observed peptide binding into DPPG monolayer from NR. In contrast, the dynamic binding process of the peptide to the zwitterionic DPPC monolayer was much lower. NR was able to relevel the structural difference of the peptide bound into the two lipid monolayers and majority of 
the peptides were associated with the charged head groups causing a significant extension of the thickness of the lipid film. These results together form a useful basis for us to develop more elaborate lipid models to characterize how these AMPs interact with model cell membranes representing different cell types, leading to better molecular models for screening the selective actions between antibacterial efficacy and host toxicity.

Supporting material contains the $\mathrm{G}_{4}$ wheel projection, more information about the surface pressure measurements, and more information about the neutron reflectivity measurements including a table of SLD values and a comparison of the three approached used for low Q data analysis and the two layer model fits for the peptide/lipid systems.

\section{Author information}

Daniela Ciumac, Biological Physics Laboratory, School of Physics and Astronomy, University of Manchester, Oxford Road, Schuster Building, Manchester M13 9PL, UK. Email: daniela.ciumac@manchester.ac.uk

Dr Richard Campbell, Institute of Laue Langevin, 71 avenue des Martyrs, CS-20156, 38042 Grenoble, France. Email: campbell@ill.eu

Professor Hai Xu, Centre for Bioengineering and Biotechnology, China University of Petroleum, Qingdao, China. Email: xuh@upc.edu.cn

Dr Luke Clifton, ISIS Neutron Facility, STFC, Chilton, Didcot OX11 0QZ, UK. Email: luke.clifton@stfc.ac.uk

Dr Arwel Hughes, ISIS Neutron Facility, STFC, Chilton, Didcot OX11 0QZ, UK. Email: arwel.hughes@stfc.ac.uk

Dr John Webster, ISIS Neutron Facility, STFC, Chilton, Didcot OX11 0QZ, UK. Email: john.webster@stfc.ac.uk

Professor Jian R Lu, Biological Physics Laboratory, School of Physics and Astronomy, University of Manchester, Oxford Road, Schuster Building, Manchester M13 9PL, UK. Email: j.lu@manchester.ac.uk

Corresponding author: Jian R Lu (email: j.lu@manchester.ac.uk; tel: +44 161 2003926)

Notes: The authors declare no competing financial interest.

\section{Author Contributions}

DC: performance research, analysed data and drafted the paper. RC: research design, data analysis and neutron experiment. LC: research design and paper writing. AH: research design and paper writing. HX: research design and knowledge of the peptide. JW: Research plan and writing. JL: research design and plan and paper writing. 


\section{Acknowledgements}

The authors acknowledge support from a Marie Curie Fellowship ITN grant (grant number 608184) under SNAL (Small nano-objects for alteration of lipid-bilayers). We also thank Dr Tom Arnold from Diamond Light Source and Dr Mario Campana at ISIS Neutron Facility, STFC for training and support. We also acknowledge the award of the beam time on FIGARO from the ILL (DOI: 10.5291/ILL-DATA.9-13-611) and thank Simon Wood for technical assistance during the experiment.

\section{References}

(1) F.C. Tenover, Am. J. Med. 119 (2006) 3-10.

(2) H.C. Neu, Science 257 (1992) 1064-1073.

(3) S.B. Levy, Sci. Am. 278 (1998) 46-53.

(4) M.C. McManus, Am. J. Health Syst. Pharm. 54 (1997) 1420-1433.

(5) D.R. Storm, K.S. Rosenthal, P.E. Swanson, Annu. Rev. Biochem. 46 (1977) 723-763.

(6) F. Schweizer, Eur. J. Pharmacol. 625 (2009) 190-194.

(7) G. Bell, P.H. Gouyon, Microbiology 149 (2003) 1367-1375.

(8) M. Dathe, T. Wieprecht, Biochim. Biophys. Acta 1462 (1999) 71-87.

(9) M.D. Lad, F. Birembaut, L.A. Clifton, R.A. Frazier, J.R. Webster, R.J. Green, Biophys. J. 92 (2007) 3575-3586.

(10) L. Silvestro, K. Gupta, J.N. Weiser, P.H. Axelsen, Biochemistry 36 (1997) 11452-11460.

(11) W. Wang, D.K. Smith, K. Mouldin, H.M. Chen, J. Biol. Chem. 273 (1998) 27438-27448.

(12) S. Frey, L.K. Tamm, Biophys. J. 60 (1991) 922-930.

(13) M. Eeman, M. Deleu, Biotechnol. Agron. Soc. Environ. 14 (2010) 719-736.

(14) B. Christensen, J. Fink, R.B. Merrifield, D. Mauzerall, Proc. Natl. Sci. USA. 85 (1988) 50725076.

(15) H. Sato, J.B. Feix, Biochim. Biophys. Acta 1758 (2006) 1245-1256.

(16) P.Wadhwani, R.F. Epand, N. Heidenreich, J. Burck, A.S. Ulrich, R.M. Epand, Biophys. J. 103 (2012) 265-274.

(17) A. Giangaspero, L. Sandri, A. Tossi, Eur. J. Biochem. 268 (2001) 5589-5600.

(18) R.E.W. Hancock, H.G. Sahl, Nat. Biotechnol. 24 (2006) 1551-1557.

(19) R.M. Epand, Biochim. Biophys. Acta 1462 (1999) 11-28.

(20) J. Hu, C. Chen, S. Zhang, X. Zhao, H. Xu, X. Zhao, J.R. Lu, Biomacromolecules 12 (2011) 3839-3843.

(21) C. Chen, J. Hu, P. Zeng, F. Pan, M. Yaseen, H. Xu, J.R. Lu, Biomaterials 35 (2014) 15521561.

(22) C. Chen, Y. Chen, C. Yang, P. Zeng, H. Xu, F. Pan, J.R. Lu, ACS Appl. Mater. Interface 7 (2015) 17346-17355.

(23) R. Maget-Dana, Biochim. Biophys. Acta 1462 (1999) 109-140. 
(24) L.A. Clifton, M.A. Sanders, A.V. Hughes, C. Neylon, R.A. Frazier, R.J. Green, Phys. Chem. Chem. Phys. 13 (2011) 17153-17162.

(25) D. Vollhardt, Adv. Colloid Interface Sci. 64 (1996) 143-171.

(26) F. Foglia, G. Fragneto, L.A. Clifton, M.J. Lawrence, D.J. Barlow, Langmuir 30 (2014) 91479156.

(27) S. Castano, B. Desbat, Biochim. Biophys. Acta 1715 (2005) 81-95.

(28) R.A. Campbell, H.P. Wacklin, I. Sutton, R. Cubitt, G. Fragneto, Eur. Phys. J. Plus. 126 (2011) 107.

(29) A. Nelson, J. Appl. Crystallogr. 39 (2006) 273-276.

(30) M. Born, E. Wolf, Principles of Optics, Pergamon Press, Oxford, 1970.

(31) Á. Ábraham, R.A. Campbell, I. Varga, Langmuir 29 (2013) 11554-11559.

(32) R.A. Campbell, A. Tummino, B. A. Noskovc, I. Varga, Soft Matter 12 (2016), 5304-5312.

(33) L. Picas, C. Suarrez-Germa, T. Montero, O. Domenech, J. Hernandez-Borrell, Langmuir 28 (2012) 701-706.

(34) I. Knyght, L. Clifton, Y. Saaka, M.J. Lawrence, D.J. Barlow, Langmuir 32 (2016) 7403-7410.

(35) M.E. Mariani, M.E. Sánchez-Borzone, D.A. García, Chem. Phys. Lipids 198 (2016) 39-45.

(36) M. Majerowicz, A.J. Waring, S. Wen, F. Bringezu, J. Phys. Chem. B 111 (2007), 3813-3821.

(37) M. Saleem, M.C. Meyer, D. Breitenstein, H-J. Galla, J. Biol. Chem. 283 (2008), 5195-5207.

(38) C-C. Lee, Y. Sun, S. Qian, H.W. Huang, Biophys. J. 100 (2011) 1688-1696.

(39) M-T. Lee, T-L. Sun, W-C. Hung, H.W. Huang. Proc. Nat. Am. Sci. 110 (2013) 14243-14248.

(40) C. Chen, J. Hu, C. Yang, Y. Zhang, F. Wang, Q. Mu, F. Pan, H. Xu, J.R. Lu, J. Mater. Chem. B 4 (2016) 2359-2368.

(41) F. Neville, M. Cahuzac, O. Konovalov, Y. Ishitsuka, K.Y.C. Lee, I. Kuzmenko, G.M. Kale, D. Gidalevitz, Biophys. J. 90 (2006) 1275-1287. 


\section{Graphical abstract}

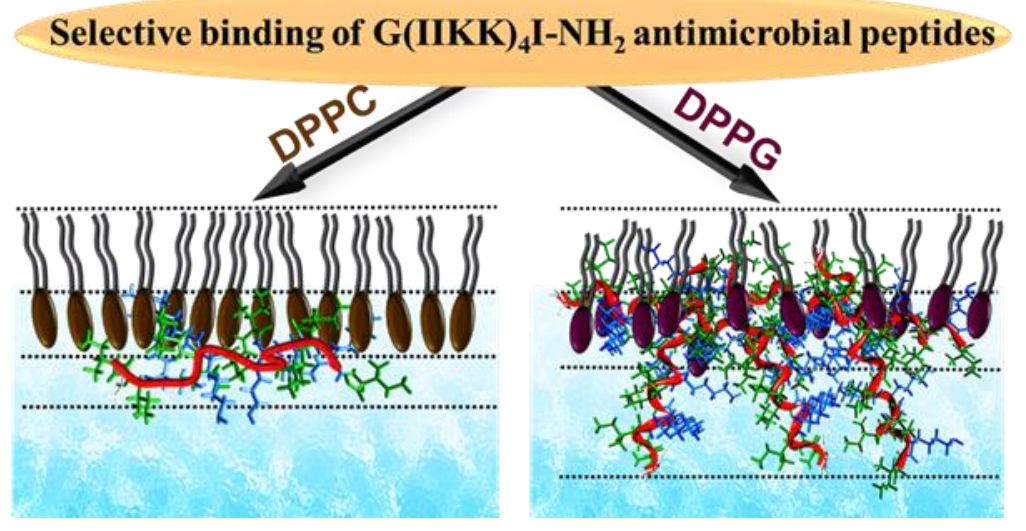

Influence of membrane surface charges on the selective binding of antimicrobial peptide G4 (G(IIKK) ${ }_{4} \mathrm{I}-$ $\mathrm{NH}_{2}$ ), where DPPC stands for 1,2-dipalmitoyl-sn-glycero-3-phosphocholine and DPPG for 1,2-dipalmitoylsn-glycero-3-phospho-(1'-rac-glycerol) (sodium salt). 